\title{
BMJ Open Effect of psychosocial work factors on the risk of certified absences from work for a diagnosed mental health problem: a protocol of a systematic review and meta-analysis of prospective studies
}

Caroline S Duchaine, ${ }^{1,2}$ Mahee Gilbert-Ouimet, ${ }^{1}$ Karine Aubé, ${ }^{1}$ Michel Vezina, ${ }^{3}$ Ruth Ndjaboue, ${ }^{1}$ Xavier Trudel, ${ }^{1,2}$ Alain Lesage, ${ }^{4}$ Lynne Moore, ${ }^{2}$ Danielle Laurin,,${ }^{1,5}$ Chantal Brisson ${ }^{1,2}$

To cite: Duchaine CS, GilbertOuimet M, Aubé K, et al. Effect of psychosocial work factors on the risk of certified absences from work for a diagnosed mental health problem: a protocol of a systematic review and meta-analysis of prospective studies. BMJ Open 2018;8:e25948. doi:10.1136/ bmjopen-2018-025948

- Prepublication history for this paper is available online To view these files, please visit the journal online (http://dx.doi org/10.1136/bmjopen-2018025948).

Received 9 August 2018 Revised 17 August 2018 Accepted 20 August 2018

D) Check for updates

(C) Author(s) (or their employer(s)) 2018. Re-use permitted under CC BY-NC. No commercial re-use. See rights and permissions. Published by BMJ.

For numbered affiliations see end of article.

Correspondence to Dr Caroline S Duchaine; caroline.duchaine@ crchudequebec.ulaval.ca

\section{ABSTRACT}

Introduction Mental health problems (MHPs) are frequent and disabling and are the first or second leading cause of certified sickness absences from work in industrialised countries. They are generally long lasting and generate a considerable human and socioeconomic burden. The deleterious effect of adverse psychosocial work factors on MHP has been documented. However, the evidence regarding the effect of these factors on absences from work for an MHP has not been synthesised since 2007. The proposed systematic review aims to synthesise the effect of adverse psychosocial work factors from three validated theoretical models (the demand-control-support, effortreward-imbalance and organisational justice models) on the risk of certified absences from work for diagnosed MHP among workers.

Method and analysis A systematic search strategy will be conducted in seven databases: Medline, Embase, CINAHL, Web of Science, Psyclnfo, Sociological abstracts and IBSS. Following Preferred Reporting Items for Systematic Reviews and Meta-Analyses recommendations, a multistep screening process by independent reviewers will lead to study selection. The search strategy was first run in 16 January 2017 and will be updated in October 2018. Only quantitative, prospective studies evaluating the effect of at least one psychosocial work factor from the validated theoretical models on certified absence from work for a diagnosed MHP will be considered for inclusion. Extracted data will be used for quantitative and qualitative evidence synthesis as well as to assess risk of bias and methodological quality. Meta-estimates will be provided for high-quality studies and by each psychosocial work factor, after considering homogeneity and number of studies.

Ethics and dissemination As this study will be based only on published studies, ethics approval is not required. Given that psychosocial works factors are frequent and modifiable, the results of this systematic review may provide evidence to support prevention strategies that can help to reduce the human social and economic burden associated with medically certified absences from work for an MHP.

PROSPERO registration number CRD42018091632.
Strengths and limitations of this study

- This will be the first systematic review with a rigorous assessment of the quality of prospective studies on the effects of psychosocial work factors on the risk of certified absences from work for a diagnosed mental health problem.

- Seven common electronic databases with the addition of two grey literature databases will be consulted following a rigorous selection process, as recommended by the Preferred Reporting Items for Systematic Reviews and Meta-Analyses statements.

- The quality of included studies will be evaluated using a validated tool specifically developed to assess the Risk of Bias in Non-randomised Studies-Interventions.

- Meta-estimates will be provided for high-quality studies and by each psychosocial work factor in accordance with heterogeneity criteria.

- As psychosocial work factors could be evaluated by several different tools, heterogeneity in the pooled data may be a limitation of this study, however, the quality assessment of these tools will help to overcome this limitation.

\section{BACKGROUND}

Mental health problems (MHPs) are a major cause of disability worldwide. Their high prevalence, long duration and high risk of recurrence place a considerable burden on the health system ${ }^{1}$ and are a major source of lost productivity for employers. ${ }^{2-4}$ In industrialised countries, MHPs are the first or second leading cause of medically certified absences from work. ${ }^{256}$ The cost of lost productive time due to depression, a major cause of absence ${ }^{7}$ and disability, ${ }^{4}$ has been estimated at US $\$ 44$ billion per year in the USA. ${ }^{8}$

There is a growing body of evidence that adverse psychosocial work factors contribute 
to the development of MHP. ${ }^{9-12}$ Adverse psychosocial work factors are defined on the basis of three validated and recognised theoretical models, the demand-controlsupport (DCS) model ${ }^{13-15}$ the effort-reward -imbalance (ERI) model $^{16}{ }^{17}$ and the organisational justice (OJ) model. ${ }^{18}$ According to the DCS model, ${ }^{14}$ high psychological demands combined with low decision latitude at work, a state known as job strain, causes psychological and physiological stress that can lead to the development of health problems. A third component, low social support at work, ${ }^{19}$ can also contribute to the development of health problems, particularly if combined with job strain. ${ }^{1920}$ The ERI model ${ }^{1617}$ postulates that an imbalance between the efforts invested at work and the rewards obtained can also lead to psychological and physiological stress, thus promoting the development of health problems. According to the OJ model, ${ }^{18}$ perceived injustice or unfairness at work can have an impact on health. ${ }^{21}$ This model refers to the equity in the rules and social norms that govern companies, particularly in terms of resources and benefits distribution (or distributive justice), the processes and procedures conditioning that distribution (or procedural justice) and interpersonal relationships (or relational justice) ${ }^{18}$ Approximately 20\%-25\% of workers in industrialised countries are exposed to adverse psychosocial work factors. ${ }^{22}$

The effect of adverse psychosocial work factors from these validated models on absences from work for MHP has been evaluated in prospective studies, ${ }^{523-29}$ but the evidence has not been synthesised in a systematic review and meta-analysis since $2007 .{ }^{30}$ Moreover, the 2007 systematic review had some limitations including no assessment of potential gender differences, no systematic analysis of the quality and/or risk of bias, and no clear definition of the MHP that caused sickness absences from work. Thus, a recent, rigorous and comprehensive systematic review is needed in order to provide an improved and updated overview of the effect of psychosocial work factors on certified absences from work for MHP. Given that psychosocial works factors are frequent and modifiable, ${ }^{31-37}$ the results of the proposed systematic review will orient evidence-based prevention strategies that are necessary to reduce the social and economic burden associated with absences due to MHP.

The aim of this study is to evaluate and synthesise the evidence of the effect of adverse psychosocial work factors from the DCS, ERI and OJ models on certified absences from work for diagnosed MHPs among workers.

\section{METHODS}

This review protocol is written in compliance with Preferred Reporting Items for Systematic Reviews and Meta-Analyses-Protocols (PRISMA-P) review guidelines ${ }^{38}$ and was registered in PROSPERO.

\section{Specific considerations}

This review is part of a broader systematic review on the effect of psychosocial work factors on all MHPs. Considering the fair amount of studies available on this topic, the review was split into two systematic reviews based on two outcomes that present important differences, namely (1) certified absences from work for a diagnosed MHP and (2) depression assessed using both self-reported and objective tools. The information sources and the electronic search strategy presented here refer to the broader review. The eligibility criteria, study selection and data extraction applied during the full-text screening refer only to the current review presented here on certified absences from work for a diagnosed MHP.

\section{Eligibility criteria}

Study design

Only original, prospective longitudinal studies will be considered. There is no restriction regarding the minimum follow-up time, however, follow-up time will be considered in the quality assessment. Case-control studies using a longitudinal design will be included, but will be subject to a separate analysis because a recall bias is possible with exposures assessed via self-report in retrospective case-control studies. Cross-sectional, qualitative and intervention studies will be excluded.

\section{Population}

The population targeted by this systematic review includes all working adults, men and women. We will not exclude studies based on country of origin, the type of job or the age and sex of participants. Studies involving only ill participants will not be considered to avoid reverse causality bias. For example, return-to-work studies of workers on sickness absences will be excluded. Studies of pregnant women will also be excluded because of pregnancy-related characteristics that may confound the association between psychosocial work factors and MHP.

\section{Exposure (intervention)}

To be included, studies must have evaluated at least one psychosocial work factor from (or similar to) the DCS (Karasek), ${ }^{13-15}$ the ERI (Siegrist) ${ }^{1617}$ or the OJ (Moorman $)^{18}$ models, using a validated instrument. The psychosocial work factors of these models are: psychological work demands, job control, social support (from colleagues and/or supervisors), efforts at work, rewards as well as procedural, distributive and relational justice. Combinations from the theoretical models will also be included: job strain (high psychological demands combined with low control), isostrain (job strain combined with low social support) and ERI (ratio between efforts and rewards). A list of known validated instruments to measure these factors was developed a priori by the research team (table 1) in order to help determine inclusion of the studies in this review. This list will be augmented during full-text readings if other tools are used following similar theoretical frameworks as the 
Table 1 Well-known validated tools used to measured psychosocial work factors

\begin{tabular}{lll}
\hline Tool & Reference for items of the questionnaires & $\begin{array}{l}\text { References of } \\
\text { validation }\end{array}$ \\
\hline Job Content Questionnaire & http://www.jcqcenter.org/ & 141519 \\
Effort-reward-imbalance questionnaire & $46-49$ & 161750 \\
Copenhagen Psychosocial Questionnaire & https://www.copsoq-network.org/ & 51 \\
& http://www.arbejdsmiljoforskning.dk/en/projekter/amis- \\
Job demands-resources & spoergeskema-om-psykisk-arbejdsmiljoe/kontaktinfo & 5253 \\
\hline
\end{tabular}

three models above and having documented evidence of validity.

\section{Comparison group}

The comparison group must be (1) from the same study population and (2) a group of workers not exposed to the psychosocial work factors mentioned above.

\section{Outcome}

Certified absence from work for a diagnosed MHP over the course of follow-up is defined as an absence from work for which there is a medical diagnosis delivered by a physician. Such objective data may be derived from employer's files, an insurance registry or a medico-administrative database. Absences for any cause and/ or self-reported absences for MHP will be excluded. As psychosocial work factors are generally assessed using self-reported methods, the use of objective indicators for the outcome greatly minimises the risk of the common method bias. ${ }^{39}$

\section{Other review eligibility criteria}

The included studies must have been published in 1979 or later, which is the year the DCS model was published, the oldest model considered in our review. Although the year will not be included in the search criteria, we will order the results by date of publication and will stop the selection in 1978. Further, only studies with original results will be included, whether they are published as original articles, book chapters or theses. No language restrictions will be applied. During the full-text screening, potentially relevant articles written in a language other than English or French will be translated by a professional translation service.

\section{Information sources}

The following electronic bibliographical databases will be searched: Medline (Ovid), Embase, CINAHL, Web of Science, PsycInfo (Ovid), Sociological abstracts and IBSS. The search strategy was first run in 16 January2017 and will be updated in October 2018. In addition to electronic databases, we will manually search in all references of (1) selected articles, (2) systematic reviews, (3) narrative reviews, (4) book chapters and (5) commentaries pertinent to this subject in order to identify potential articles for inclusion. We will also consult two international grey literature databases: The Grey Literature Report from the
New York Academy of Medicine, which specialises in articles related to public health, as well as WHO-IRIS, which contains all the reports of WHO.

\section{Search strategy}

The search strategy will combine four sets of keywords customised to each database (ie, Medical Subheading terms where appropriate) pertaining to (1) Population (workers); (2) Exposure (psychosocial work factors from the DCS, ERI and OJ models); (3) Concepts and terms that refer to both the population and the exposure (work stress); (4) Outcome (all MHP including certified absences for MHP). The searches will be run again just before the final analyses in order to identify studies published after our search date for possible inclusion in our review. There will be no date, design or language restrictions.

An example of the search strategy performed for Medline (Ovid) on 31 October 2017 is provided in table 2. As mentioned previously, this search strategy is more inclusive in order to cover all aspects of the broader systematic review on psychosocial work factors and all MHP.

\section{Study records}

Data management

The bibliographical citations and abstracts from the seven databases, identified using the search strategy, will be pooled into the reference management software Endnote. Duplicates will be removed using the automatic function in EndNote in addition to manual elimination during screening. Screening by title and abstract will be done using Endnote and full-text screening will be conducted using Excel. A screening form with the inclusion and exclusion criteria will be developed and tested. All members of the screening team will be trained on how to use Endnote, Excel and the screening form before participation.

\section{Selection process}

The titles and abstracts of the studies will be screened independently by two reviewers (CSD and KA). During the screening by title and abstract, the references will be classified into four groups: (1) YES: all the eligibility criteria were met, (2) NO: at least one of the criteria was clearly not met, (3) UNCLEAR: the eligibility cannot be 
Table 2 Search strategy for Medline (Ovid) carried out on 31 October 2017

\begin{tabular}{|c|c|c|c|c|c|}
\hline \multirow[b]{2}{*}{ \#Line } & \multirow[b]{2}{*}{ Set of keywords } & \multirow[b]{2}{*}{ Search strategy } & \multirow[b]{2}{*}{ Results } & \multicolumn{2}{|c|}{ Combination of keywords } \\
\hline & & & & \#Line & Total \\
\hline$\# 1$ & $\begin{array}{l}\text { Concepts and terms } \\
\text { that refer to both the } \\
\text { population and exposure }\end{array}$ & $\begin{array}{l}\text { (job adj2 (control OR security OR insecurity OR } \\
\text { strain OR stress OR stressor OR stressors OR } \\
\text { demand OR demands OR demanding)).tw. OR } \\
\text { *workload/OR workload.tw. OR (work adj2 (stress } \\
\text { OR stressor OR stressors)).tw. }\end{array}$ & 35611 & $\begin{array}{l}\text { \#5: } \\
\text { \#1 AND \#4 }\end{array}$ & 4331 \\
\hline
\end{tabular}

\#3 Exposure: Psychosocial work factors from the demand-controlsupport, effortreward-imbalance and organisational justice models

exp *sociological factors/px OR *stress,

Psychological/OR *social justice/OR *reward/ or reward.tw. OR overcommitment.tw. OR siegrist*. tw. OR siegrist j.au. OR karasek*.tw. OR karasek r.au. OR karasek ra.au. OR skill discretion.tw. OR social support.tw. OR * Social Support/OR (isostrain OR iso strain).tw. OR decision authority. tw. OR decision making.tw. OR *Decision Making/ OR (decision adj2 latitude*).tw. OR (demand* adj2 latitude*).tw. OR (demand* adj2 control).tw. OR (psychological $^{*}$ adj2 demand ${ }^{*}$ ).tw. OR (equity or inequities or inequity).tw. OR (intrinsic adj5 effort*). tw. OR psychosocial.tw. OR ((organizational OR organisational OR distributive OR procedural OR interactional OR relational) adj5 (justice OR injustice)).tw.

\section{\#4 Outcomes:}

(Psychiatric* adj2 morbidity).tw. OR

Mental health problems (MHP), including medically certified sickness absences for MHP

Hospitalisation*.tw. OR *Hospitalization/OR Hospitalization*.tw. OR exp *Somatoform Disorders/ OR (((leave OR leaves OR absence) and (disability OR sickness OR sick)) OR sick-list*).tw. OR sick leave/ORAbsenteeism.tw. OR *Absenteeism/OR psychotrop*.tw. OR exp *Psychotropic Drugs/ OR (Psychological disorder OR Psychological disorders).tw. OR *Psychophysiologic Disorders/ ep OR *Psychophysiologic Disorders/pp OR *Psychophysiologic Disorders/px OR (Mental disorder OR Mental disorders).tw. OR Mental health.tw. OR *Mental Health/OR Anxiolytic*.tw. OR Anxiety.tw. OR Antidepress*.tw. OR *Anxiety/ ORdepressive.tw. OR *Depressive Disorder/ OR *Depression/or depression.tw. OR *Anxiety Disorders/OR *Mental Disorders/ep OR *Mental Disorders/pp OR *Mental Disorders/px

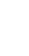

(1)


other reviewers. Kappa will be calculated between each pair of reviewers. Results will be compared and disagreements will be resolved by discussion or by the opinion of the principal investigator of the research team (CB) if consensus is not reached. The list of references of included articles as well as systematic or narrative literature reviews, book chapters, commentaries, editorials or letters will be revised to identify articles that may have been missed.

\section{Data collection process}

A standardised extraction grid containing all the data items listed in the following section will be used for data extraction. The grid will be tested by two independent reviewers (CSD and KA) with five studies and will be improved for the complete extraction process. Data extraction will be performed in duplicate by three independent reviewers (CSD, KA and one research assistant). The corresponding authors of retained articles may be contacted to request any missing information required for the completion of the extraction grid. Discrepancies between the three reviewers will be resolved through discussion, and with the principal investigator of the research team $(\mathrm{CB})$ when necessary.

\section{Data items}

Extracted data will include: (1) study population (cohort name, year of study, country, sample size, participation and attrition rates, mean age, gender, socioeconomic status); (2) study design (prospective, case control, duration of follow-up); (3) type of employment (white-collar workers, blue-collar, self-employed, manual, office, etc); (4) psychosocial work factors (name, questionnaire used, validation, language translation validation, categorisation, timing of exposure, assessment of duration of exposure); (5) certified absence from work for a diagnosed MHP (source of data (obtained from employer, administrative or insurance files), diagnostic codes, MHP eg, major depressive disorder, generalised anxiety disorder, etc); (6) analysis (types of models used, confounding variables included, treatment of missing data and losses at follow-up, sensitivity analyses, gender-stratified analyses, interaction tests, etc); (7) results (number of sickness absences, prevalence of exposure, effect measures with confidence intervals for (i) each analysis performed, (ii) each psychosocial work factor considered and (iii) each mental health outcome studied) and (8) potential limitations mentioned by the authors (selection bias $(\%$ participation at baseline and at follow-up, missing data), confounding bias (confounding variables taken into account), information bias (measurement of exposure and/or outcome) and lack of statistical power).

\section{Risk of bias and confidence in cumulative evidence}

The risk of bias and the quality level for each included study will be evaluated in duplicate by four independent reviewers (CB, CSD, MG-O and XT). Each reviewer will have article in common with all the other reviewers. The
Risk of Bias in Non-randomised Studies-Interventions (ROBINS-I) tool, specifically developed to assess bias in non-randomised studies, ${ }^{40}$ will be used. This tool was developed over 3 years by expert consensus in collaboration with the Cochrane Review Groups. They identified seven domains in which bias might be introduced into non-randomised studies. These domains include: (1) baseline and time varying confounding, (2) selection bias attributable to exclusion of eligible participants or loss at follow-up, (3) differential or non-differential misclassification of exposure of interest, (4) bias due to change of exposure groups for some participants, (5) bias due to missing data, (6) differential or non-differential misclassification of the outcome and (7) bias introduced by selective reporting of results in a way that depends on the findings. Within each domain, the evaluator answers several questions in order to assess the level of risk of bias on a four-level scale (low, moderate, serious and critical risk of bias). The ROBINS-I tool includes a template and a detailed user guide. Other quality criteria relevant for studies on the specific topic of the current review will also be included in the quality assessment procedure. These include the temporal dimension of exposure and outcome (duration and timing of exposure) and the potential for overadjustment. The risk of bias will allow us to assess the methodological quality of included studies and this information will be summarised. For data synthesis, this assessment will allow us to examine the effect of study quality on the findings of this review.

\section{Data synthesis}

All included studies will be synthesised in a table for quantitative and qualitative evaluation. We will provide a narrative and quantitative synthesis of the findings. Pooled estimates will be calculated separately for the different psychosocial work factors. The choice of the type of model (fixed or random) will be based on the heterogeneity of included studies. Heterogeneity will be tested using $\mathrm{I}^{2}$, which quantifies the percentage of variability between study results due to real difference between groups rather than at random. ${ }^{41}{ }^{42}$ In the event of $\mathrm{I}^{2} \geq 0.50$ (high heterogeneity), random models will be used because of their more conservative nature (CIs tend to be wider).$^{41} \mathrm{I}^{2}$ will also be used to compare heterogeneity across different subgroups of studies assessed separately (eg, higher quality studies compared with all studies). According to our knowledge of the literature, we estimate that, for each of the three models considered (DCS, ERI and OJ), the available studies will be sufficient in number $(n \geq 3)$ to produce global meta-estimates as well as meta-estimates according to: (1) methodological quality and (2) gender.

Meta-regressions will also be performed to examine variation in relative risks according to individual studies' characteristics. These will model the log risk ratio for each psychosocial work factor as a function of the following explanatory variables: gender, risk of bias, duration of exposure and adjustment for confounders. For the latter, the potential overadjustment for mediating variables 
will be examined using separate models. Random-effect meta-regression will be used to consider the residual heterogeneity not modelled by the explanatory variables.

\section{Meta-bias}

The presence of a publication bias will be verified visually with funnel plot graphs as well as the Egger statistical test. This analysis assesses whether studies with a large sample size are more likely to detect lower effect measures than studies with a small sample size. Separate graphs will be produced based on: (1) models of psychosocial work factors and (2) effect measures observed in women and men. The reporting technique of this systematic review will comply with PRISMA review guidelines. ${ }^{43}$

\section{Dissemination}

The result will be published in a peer-review journal. The proposed systematic review is essential to support prevention strategies to reduce absences from work due to MHP. This review will provide evidence that could help physicians identify factors contributing to their patients' MHP ${ }^{44}$ support workplace decision-makers to improve the psychosocial work environment ${ }^{35-37}$ and support public policy-makers to improve national standards, such as the Canadian National Standard for Psychological Healthy and Safety in the Workplace. ${ }^{45}$

\section{Patient and public involvement}

Patients and public were not involved in the development of the research question and in the design of this study.

\section{Author affiliations}

${ }^{1}$ Population Health and Optimal Health Practices Unit, CHU de Québec Research Center, Quebec, Canada

${ }^{2}$ Faculty of Medicine, Laval University, Quebec, Canada

${ }^{3}$ Institut National de Santé Publique du Québec, Quebec, Canada

${ }^{4}$ Department of Psychiatry, Montréal Université, Montréal, Canada

${ }^{5}$ Faculty of Pharmacy, Laval University, Quebec, Canada

Acknowledgements The authors want to thank the Canadian Institute of Health Research for financial support.

Contributors $C B$ is the principal investigator of the study. With the close contribution of MG- 0 , she planned and designed this study protocol and revised the first draft. CSD wrote the first draft of the manuscript. KA contributed to the first draft of the manuscript and revised the final version. $\mathrm{CB}, \mathrm{XT}, \mathrm{MG}-0, \mathrm{RN}$ and MV bring expertise in the definition of psychosocial work factors. MV prepared the dissemination plan. MG- 0 brings expertise in gender differences. AL and RN bring expertise in the definition of mental health problems and economic statistics. LM brings expertise in the design of systematic reviews and meta-analyses. DL supervised the writing of the manuscript and brings expertise in systematic reviews. All the authors revised and approved the final version of this manuscript.

Funding This protocol was supported by the Canadian Institute of Health Research (CIHR grant number\#201404KRS-329015-KRS-CFBA-35698).

Competing interests None declared.

Patient consent Not required.

Ethics approval As this study will be based only on published studies, ethics approval is not required.

Provenance and peer review Not commissioned; peer reviewed for ethical and funding approval prior to submission.

Open access This is an open access article distributed in accordance with the Creative Commons Attribution Non Commercial (CC BY-NC 4.0) license, which permits others to distribute, remix, adapt, build upon this work non-commercially, and license their derivative works on different terms, provided the original work is properly cited, appropriate credit is given, any changes made indicated, and the use is non-commercial. See: http://creativecommons.org/licenses/by-nc/4.0/.

\section{REFERENCES}

1. Lim KL, Jacobs $P$, Ohinmaa A, et al. A new population-based measure of the economic burden of mental illness in Canada. Chronic Dis Can 2008;28:92-8.

2. Henderson M, Glozier N, Holland Elliott K. Long term sickness absence. BMJ 2005;330:802-3.

3. Koopmans PC, Bültmann U, Roelen CA, et al. Recurrence of sickness absence due to common mental disorders. Int Arch Occup Environ Health 2011;84:193-201.

4. Whiteford HA, Degenhardt L, Rehm J, et al. Global burden of disease attributable to mental and substance use disorders: findings from the Global Burden of Disease Study 2010. Lancet 2013;382:1575-86.

5. Bourbonnais R, Brisson C, Vézina M, et al. Psychosocial work environment and certified sick leave among nurses during organizational changes and downsizing. Relations industrielles 2005;60:483-509.

6. Black DC, Frost D. Health at work - an independent review of sickness absence. London: Parliament, Secretary of State for Work and Pensions, 2011.

7. Koopmans PC, Roelen CA, Groothoff JW. Sickness absence due to depressive symptoms. Int Arch Occup Environ Health 2008;81:711-9.

8. Stewart WF, Ricci JA, Chee E, et al. Cost of lost productive work time among US workers with depression. JAMA 2003;289:3135-44.

9. Bonde JP. Psychosocial factors at work and risk of depression: a systematic review of the epidemiological evidence. Occup Environ Med 2008;65:438-45.

10. Stansfeld S, Candy B. Psychosocial work environment and mental health-a meta-analytic review. Scand J Work Environ Health 2006;32:443-62.

11. Theorell $\mathrm{T}$, Hammarström A, Aronsson G, et al. A systematic review including meta-analysis of work environment and depressive symptoms. BMC Public Health 2015;15:738.

12. Netterstrøm B, Conrad N, Bech P, et al. The relation between workrelated psychosocial factors and the development of depression. Epidemiol Rev 2008;30:118-32.

13. Karasek RA. Job demands, job decision latitude, and mental strain: Implications for job redesign. Adm Sci Q 1979;24:285-308.

14. Karasek R, Brisson C, Kawakami N, et al. The Job Content Questionnaire (JCQ): an instrument for internationally comparative assessments of psychosocial job characteristics. J Occup Health Psychol 1998;3:322-55.

15. p.Karasek R, Theorell T, stress Hwork. productivity and reconstruction of working life. New York: Wiley 1990:381.

16. Siegrist J. Adverse health effects of high-effort/low-reward conditions. J Occup Health Psychol 1996;1:27-41.

17. Siegrist J, Starke D, Chandola T, et al. The measurement of effortreward imbalance at work: European comparisons. Soc Sci Med 2004;58:1483-99.

18. Moorman RH. Relationship between organizational justice and organizational citizenship behaviors: Do fairness perceptions influence employee citizenship? J Appl Psychol 1991;76:845-55.

19. Johnson JV. Theoretical developments in psychosocial work environment research. Int J Health Serv 1989;19:457-8.

20. Eller NH, Netterstrøm B, Gyntelberg F, et al. Work-related psychosocial factors and the development of ischemic heart disease: a systematic review. Cardiol Rev 2009;17:83-97.

21. Elovainio M, Kivimäki M, Vahtera J. Organizational justice: evidence of a new psychosocial predictor of health. Am J Public Health 2002;92:105-8.

22. Brisson C, Aboa-Eboulé C, Leroux I, et al; Psychosocial factors at work and heart disease, Heart and Mind: The Practice of Cardiac Psychology. Second edition: American Psychological Association, 2011:269-86.

23. Juvani A, Oksanen T, Virtanen M, et al. Organizational justice and disability pension from all-causes, depression and musculoskeletal diseases: A Finnish cohort study of public sector employees. Scand $J$ Work Environ Health 2016;42:395-404.

24. Bourbonnais R, Mondor M. Job strain and sickness absence among nurses in the province of Québec. Am J Ind Med 2001;39:194-202.

25. Janssens $H$, Clays $E$, De Clercq $B$, et al. The relation between psychosocial risk factors and cause-specific long-term sickness absence. Eur J Public Health 2014:24:428-33. 
26. Clumeck N, Kempenaers C, Godin I, et al. Working conditions predict incidence of long-term spells of sick leave due to depression: results from the Belstress I prospective study. J Epidemiol Community Health 2009;63:286-92.

27. Inoue A, Kawakami N, Haratani T, et al. Job stressors and longterm sick leave due to depressive disorders among Japanese male employees: findings from the Japan work stress and health cohort study. J Epidemiol Community Health 2010;64:229-35.

28. Ndjaboué R, Brisson C, Vézina M, et al. Effort-reward imbalance and medically certified absence for mental health problems: a prospective study of white-collar workers. Occup Environ Med 2014;71:40-7.

29. Mather L, Bergström G, Blom V, et al. High job demands, job strain, and iso-strain are risk factors for sick leave due to mental disorders: A prospective swedish twin study with a 5-year follow-up. J Occup Environ Med 2015;57:858-65.

30. Duijts SF, Kant I, Swaen GM, et al. A meta-analysis of observational studies identifies predictors of sickness absence. J Clin Epidemiol 2007;60:1105-15.

31. Brisson C, Gilbert-Ouimet M, Duchaine CS, et al. Workplace Interventions Aiming to Improve Psychosocial Work Factors and Related Health. In: SiegristJ, Wahrendorf M, eds. Work Stress and Health in a Globalized Economy. Switzerland: Springer, 2016:333-64.

32. Bourbonnais R, Comeau M, Vézina M. Job strain and evolution of mental health among nurses. J Occup Health Psychol 1999;4:95-107.

33. Bourbonnais R, Brisson C, Vinet A, et al. Effectiveness of a participative intervention on psychosocial work factors to prevent mental health problems in a hospital setting. Occup Environ Med 2006;63:335-42.

34. Bourbonnais $\mathrm{R}$, Comeau $\mathrm{M}$, Vézina $\mathrm{M}$, et al. Job strain, psychological distress, and burnout in nurses. Am J Ind Med 1998;34:20-8.

35. Bourbonnais R, Brisson C, Vézina M. Long-term effects of an intervention on psychosocial work factors among healthcare professionals in a hospital setting. Occup Environ Med 2011;68:479-86

36. Brisson C, Cantin V, Larocque B, et al. Intervention Research on Work Organization and Health: Research Design and Preliminary Results on Mental Health. Can J Commun Ment Health 2006;25:241-59.

37. Gilbert-Ouimet M, Brisson C, Vézina M, et al. Intervention study on psychosocial work factors and mental health and musculoskeletal outcomes. Healthc Pap 2011;11 Spec No-47-66.

38. Moher D, Shamseer L, Clarke M, et al. Preferred reporting items for systematic review and meta-analysis protocols (PRISMA-P) 2015 statement. Syst Rev 2015;4:1):1.
39. Meade AW, Watson AM, Kroustalis C. Assessing Common Methods Bias in Organizational Research. New York: 22nd Annual Meeting of the Society for Industrial and Organizational Psychology, 2007.

40. Sterne JA, Hernán MA, Reeves BC, et al. ROBINS-I: a tool for assessing risk of bias in non-randomised studies of interventions. BMJ 2016;355:i4919.

41. Higgins JP, Thompson SG, Deeks JJ, et al. Measuring inconsistency in meta-analyses. BMJ 2003;327:557-60.

42. Egger M, Smith GD, Phillips AN. Meta-analysis: principles and procedures. BMJ 1997;315:1533-7.

43. Liberati A, Altman DG, Tetzlaff $\mathrm{J}$, et al. The PRISMA statement for reporting systematic reviews and meta-analyses of studies that evaluate health care interventions: explanation and elaboration. J Clin Epidemiol 2009;62:e1-34.

44. Commission de la santé mentale du Canada. Santé et sécurité psychologiques: guide de l'employeur. $2012 \mathrm{http}: / /$ www.mentalhe althcommission.ca/SiteCollectionDocuments/Workforce/Workforce Employers Guide FRE.pdf.

45. Bureau de Normalisation du Québec, 2008. BNQ 9700-800 Prévention, promotion et pratiques organisationnelles favorables à la santé en milieu de travail. http://www.bnq.qc.ca/documents/9700800_description_norme.pdf.

46. Siegrist J, 2003. The model of effort-reward imbalance:Measurement http://www.uni-duesseldorf.de/MedicalSociology/index-eri.htm.

47. Siegrist J. Effort-reward imbalance at work - theory, measurement and evidence. Germany: University Dusseldorf, 2012. Contract No.: 17 Jun 2016

48. Siegrist J, Montano D. ERI - Questionnaires - Users Guide. Germany: University Dusseldorf, 2013. Contract No.: version 22.11.2012.

49. Siegrist J, Peter R. Measuring effort-reward imbalance at work: Guidelines. Düsseldorf, 1996.

50. Siegrist J. Adverse health effects of effort-reward imbalance at work: theory, empirical support, and implications for prevention: In. Cooper C, ed. Theories of organizational stress. Oxford: Oxford University Press, 1998:190-204.

51. Kristensen TS, Hannerz $\mathrm{H}$, Høgh A, et al. The Copenhagen Psychosocial Questionnaire-a tool for the assessment and improvement of the psychosocial work environment. Scand J Work Environ Health 2005;31:438-49.

52. Demerouti E, Bakker AB, Nachreiner F, et al. The job demandsresources model of burnout. J Appl Psychol 2001;86:499-512.

53. Bakker AB, Demerouti E. The Job Demands-Resources model: state of the art. $J$ manage psychol 2007;22:309-28. 